\title{
Preventing paraffination of pumping equipment of oil wells
}

\author{
Krasnov Andrei Nikolaevich \\ Department of Workflow and Production Automation \\ Ufa State Petroleum Technological University \\ Ufa, Russia \\ ufa-znanie@mail.ru
}

\author{
Khoroshavina Elena Aleksandrovna \\ Department of Workflow and Production Automation \\ Ufa State Petroleum Technological University \\ Ufa, Russia \\ elena_horoshavina@rambler.ru
}

\author{
Prakhova Marina Yurevna \\ Department of Workflow and Production Automation \\ Ufa State Petroleum Technological University \\ Ufa, Russia \\ prakhovamarina@yandex.ru
}

\begin{abstract}
In the overall balance of oil reserves of Russia, highparaffin oils (HWO) make up, by different estimations, from 55 to $75 \%$. Asphalt-resinous and paraffin depositions (ARPD) are contained in the composition of oils in almost all oil-producing regions of the Russian Federation (RF). High content of paraffin (more than 6\%) is one of the indicators, by which oil is related to the hard-to-recover type. The chemical composition of ARPD depends on the properties of extracted oil, thermo- and hydrodynamic conditions of productive strata, geological and physical peculiarities, the method of development and exploitation of deposits. ARPD accumulation in the air-gas channel of oil-field equipment and on the internal side of pipes leads to the reduction of the system performance, a decrease of the overhaul period and effectiveness of the operation of pump assemblies, an increase of the wear of equipment, electric energy consumption and pressure in the flowlines. In extreme cases, it can result in the well shutdown due to complete overlap of cross-section of tubing string or presence of ARPD in the well pumps. The ARPD is controlled in two directions: prevention or the slowing down of formation of sediments and removal of already formed ARPD. To prevent paraffination of the pump assembly, chemical and physical methods are used, and to remove deposited paraffin - mechanical, thermal, and chemical methods. None of the enumerated methods is free from disadvantages. To prevent depositions, it is expedient to use physical impacts, reducing coalescence of separate particles of hydrocarbonaceous associates. It is proposed using electrolysis of mineralized stratum water and creating electrostatic field with polarity, similar to ARPD particles, as such impacts. Technical realisation of these effects is possible by creation of the electrochemical chain, similar to the one used during cathode protection. Using the method provides unhindered flotation of paraffin crystals to the wellhead without their deposition on the well equipment.
\end{abstract}

Key words - asphalt-resin-paraffin depositions, ARPD, paraffination of well equipment, depositions control, electrolysis

\section{INTRODUCTION}

The majority of Russian oil-producing deposits are at the late stage of development, for which a reduction of productivity of output wells and an increase of the complicated well stock are typical [1]. After finishing the period of fountain extraction, the main means of supplying crude oil to the surface are subsurface well pumps (SWP) and submersible electric-centrifugal pumps (ECP), feeding well fluid to the wellhead through tubing string (TS) of different types and diameters.

One of the most serious problems arising during transition to the mechanized extraction and a reduction of the oil-well production rate is accumulation of so called asphalt-resinous and paraffin depositions (ARPD) on TS walls and in pump assemblies themselves. Paraffin depositions form mainly owing to precipitation (crystallisation) of high-molecular hydrocarbons when reducing the temperature of oil flow. These depositions decrease filtration characteristics of the stratum, plug the pores, reduce the useful cross-section of tubing string and, as a consequence, complicate the oil extraction considerably, increase the electric power consumption during mechanized way of extraction, lead to increased wear of equipment [2]. In addition to the reduction of efficiency of operation of pump assemblies, formation of viscous media and paraffin depositions in the output wells initiate arising emergencies, most of which are related to sucker rod and polished rod breakage, which reduces many times the overhaul period and the volume of extraction.

Formation of paraffin depositions on the TS walls is also connected with the increase of water cut of the extracted fluid and formation of stable emulsions in wells. The main reason of these manifestations is the presence of water and movement (slipping) of oil relatively water. Formation of depositions during oil extraction is enabled by an increase of the concen- 
tration of asphalt-resin-paraffin compounds on the surface of oil drops. When lifting oil with water uphole, stabilization of the surface film and its cooling take place, which is accompanied by an additional increase of viscosity of the lifted product. As a result, the surface layer acquires adhesiveness and is easily deposited due to high activity on the surface of oil-field equipment.

The most high-paraffin oils on the territory of the Russian Federation are concentrated in oil-and-gas bearing basins of the European part of Russia: Yeniseisk -Anabar, TimanPechora, Volgo-Ural and Caspian [3]. However, this problem is manifested to the fullest degree both in Western Siberia and in the North, where in comparison with other regions, the difference between stratum temperatures and the temperature in the well bore is the biggest owing to the presence of the relic zone of permafrost: in the depth interval up to $800 \mathrm{~m}$, paraffin solid precipitates form very intensively [4].

To control ARPD and consequences of its presence, there are numerous mechanical, chemical and physical methods, allowing reducing these manifestations. However, in reality, all of them are costly and are not always effective. Besides, the majority of problem wells are crooked, prone to precipitation of salts, loss of abrasive particles, have aggressive environment and a significant gas factor. These factors hinder timely diagnostics of paraffination and, as a consequence, its control.

The main direction of ARPD control when extracting oil must be their prevention. Therefore, development of new principles and methods of prevention of equipment paraffination, in particular, TS, is a relevant task, the solution of which will allow decreasing the fund of inactive wells, increasing overhaul period of their exploitation and, as a consequence, increasing the volume of extracted oil.

\section{RESULTS AND DISCUSSIONS}

\section{Basic facts about ARPD}

ARPD control is a complex engineering problem because the process of depositions' formation is extremely complex and is not studied completely [5-10]. Such physical and chemical processes can participate in it as sedimentation of mechanically suspended particles, coarsening and precipitation of dispersed components, saturation of molecular-dissolved components and formation of crystals, adsorption of components of the system, etc. Such variety of physical and chemical processes increases sharply the number of factors, influencing the process of ARPD formation. The presence of particles of sand, clay and other mechanical admixtures in oil facilitates ARPD strengthening, frequently acting as nucleation centres of paraffin. Depending on the conditions, compositions of paraffin depositions are very various even in one well. They differ by the content of asphaltenes, tars and solid hydrocarbons. A characteristic peculiarity of the process is nonuniform distribution of paraffin in the mass of depositions throughout the cross-section of the stratum. The largest amount of paraffin is contained in the stratum, which is adjacent directly to the wall. This indicates that in the course of accumulation of depositions in the inner layers, paraffins recrystallize. Depositions become denser, and liquid phase is extruded. ARPD profile forms with a constant increase of thickness towards the wellhead and depends on the TS diameter (Figure 1). Direct relation between the paraffin content and the intensity of its depositions is absent. This is explained by the fact that peculiarities of formation of paraffin depositions mainly depend not only on the absolute number of solid hydrocarbons, but also on their composition (hydrocarbon content with branched structures).

In practice, depositions represent a gel-like mixture, consisting of ARPD, stabilised water globules, mechanical admixtures and mineral salts. At that, the main material of depositions is paraffin, but resins and asphaltenes act as a cementing substance.

The mechanism "paraffination" implies a set of processes, leading to accumulation of the solid organic phase on the equipment surface [1]. Usually, the term "paraffins" combines the whole hydrocarbonaceous part of depositions. Modern ideas about the mechanism of formation of paraffin depositions on the well equipment can be conditionally subdivided into sedimentary-volumetric and crystallisation-surface theory [11]. The first theory implies that paraffin crystals form in the volume of the flowing oil, gradually precipitate on the metal surface and attach to it, forming gradually a sedimentary stratum of organic depositions. As to the second mechanism, paraffin crystals form directly on the metal surface and gradually crystalize into complexes. The process of paraffin crystallisation on the surface proceeds owing to feed from oil solution. There is also a third mixed mechanism, combining in itself all the peculiarities of the first two.

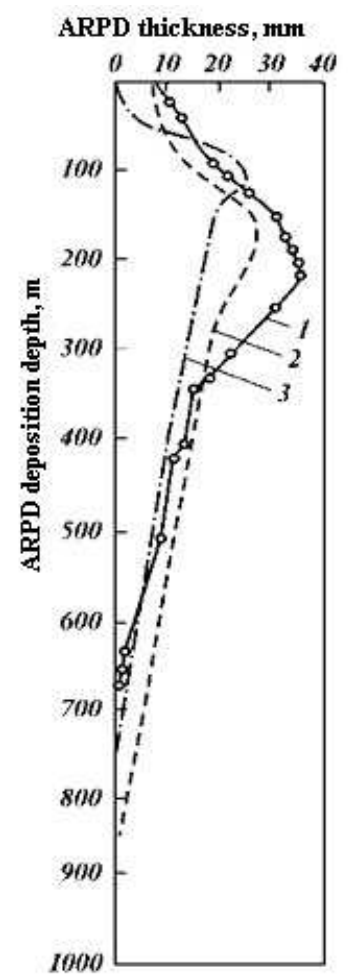

Fig. 1. Dynamics of ARPD formation along length of TS of different diameters: $1-89 \mathrm{~mm} ; 2-73 \mathrm{~mm} ; 3-62 \mathrm{~mm}$. 
At that, the condition of the surface and its nature influence considerably the process of paraffin depositions formation.

The necessary conditions of paraffin deposition formations are:

presence in oil of high-molecular hydrocarbon compounds, and, in first place - of the methane series (of paraffins);

reduction of stratum pressure up to saturation pressure;

reduction of the stream temperature up to values, by which the solid phase precipitates from oil;

presence of a substrate with lowered temperature, on which high-molecular hydrocarbons with their sufficiently strong adhesion to the surface, eliminating the possibility of tearing off depositions by the stream of the liquid-gas mixture or oil with a preset technological mode.

There are many other factors, facilitating or preventing from intensive formation of paraffin depositions.

The following factors can be named as basic [12]:

adsorption processes, occurring on the interface "solid body (metal) - paraffin" and consisting in natural properties of paraffin depositions, which include resin substances;

presence of products of stratum destruction, mechanical admixtures, introduced from the surface during technological operations, products of metals corrosion, etc. on the depositions' surfaces;

- surface roughness, being the basis for "adhesion" of paraffin crystals, around which deposition aggregates start to grow;

- the movement rate of the gas-liquid mixture, which can provide precipitation of crystals on the surface of solid bodies or, vice versa, their tearing off the surface, as well as removal to the well head;

electric-kinetic phenomena, causing electrization of both the surface of the tube wall and the surface of the paraffin crystal, which strengthens adhesion of paraffin to the metal;

- flow structure: the largest depositions appear during bubble gas-piston flow, when gas is a dispersed phase in the form of big bubbles.

- cooling of the liquid during intensive degassing of oil in TS;

- low water cut: small amount of stratum water leads to the reduction of liquid temperature owing to the reduction of heat capacity of borehole fluid and hydrophilization of the TS surface, preventing from ARPD deposition on this surface.

\section{ANALYSIS OF EXISTING WAYS OF ARPD CONTROL}

To remove ARPD, different methods that can be conditionally divided into four basic groups: mechanical, thermal, chemical and physical [13-16]:

mechanical - scrubbing different constructions with manual or mechanized hoists;

heat (thermal) - hot oil washing, hot water washing with addition of surface-active substances, heating TS with vapour or with the use of stationary installed or submersed electric heaters, warming cables, etc.;

chemical - using hydrocarbon solvents or waterbased washing compounds with addition of surface active substances for dissolving formed paraffin depositions, as well as inhibitors of paraffin depositions for preventing ARPD precipitation on TS walls; physical - use of ultrasound (stationary installed or submersed emitters), constant magnetic or highfrequency electric magnetic fields, as well as covering the inner surfaces of pipes with enamels, glasses and other materials, reducing TS roughness.

Each of the methods has a number of shortcomings in addition to advantages.

Mechanicals -scrapers and hoists - often fail; scrapers are stuck in paraffinic pipes. They cannot be applied to clean the wellbore area from paraffin and allow controlling paraffin depositions only in lifting pipes, whereas the well production rate decreases because of paraffin depositions in the manifold and the flow line. The most important thing is that during cleaning the well, it shuts down, and the duration of the operation usually amounts to $3-5$ days [12].

The main disadvantages of the thermal method include: necessity of well shutdown when conducting works, which reduces the coefficient of their exploitation; since, as a rule, highly-watered production is used from adjoining hot wells, there is probability of formation of stable emulsions;

- shortcoming of using vapour as a heat-transfer medium is that vapour, descending in the well, cools down, condensates, turning into the cooled water, which is unable to melt the formed resin-paraffin depositions at a large depth;

- the use of hot water can lead to formation of the paraffin plug at the discharge collector, since paraffin depositions, brought out from the well lift, during cooling precipitate again on the walls of the oil field equipment;

application of electric heating is hindered because of high additional electric consumptions and complications during the operation of downhole equipment, which can lead to its preliminary failures.

It is possible to note the complexity of selection of reagent for a specific well as a shortcoming of the chemical method since its composition depends on the ARPD compound, which, in its turn, is determined by the oil composition. Besides, to prevent paraffination, chemical reagents are pumped into the wellbore area or tube space, are adsorbed on the surface of stratal rocks and during development are freed into the oil flow. Such feed does not guarantee a specific and accurate dosage of the reagent into a zone of the most probable ARPD deposition [17]. This is related to the height and variations of the dynamic level, oil density in the tube space above the pump suction, presence of the degassing zone, density of the chemical reagent, its dissolution in oil, etc. At that, all reagents exert pernicious influence on the environment. 
The most effective inhibitors of ARPD (composite reagents, consisting of several chemical compounds of different chemical nature and with different mechanisms of influence on the process of ARPD strata formation on the surface of technological equipment) are very costly. Their use requires conducting additional studies of the composition of paraffin depositions and the temperature of their melting.

As to the physical methods, ultrasound heating of production inside the well requires the use of generators with very high output voltage (several kilovolt), which increases probability of emergencies in the deposit. Besides, when descending emitters into the borehole, wire breakage occurs with losses of costly equipment. When using the constant magnetic field, there are no such problems, but owing to significant sizes of magnets $(2-5 \mathrm{~m})$ and their mass $(30-100 \mathrm{~kg})$, the problems of their installation and mounting arise (lifting TS is obligatory). After magnetic processing of the borehole fluid, adhesion of formed paraffin particles is hindered; as a result, they do not precipitate on the pipe walls, but are brought with the liquid flow. The phenomenon nature remains incompletely clear and is manifested not on all ARPD [18].

\section{DESCRIPTION OF THE PROPOSED METHOD}

Despite a significant number of methods of controlling ARPD, the variety of conditions of deposit development and the difference of characteristics of the extracted production frequently requires an individual approach and even development of new technologies.

The concept of the proposed method of controlling ARPD deposition on the oil-pumping equipment in oil wells is built on the use of several physical and chemical effects, namely on a decrease of adhesion among separate particles of ARPD, local thermal influence and creation of the electrostatic field of a certain polarity.

The first two effects are created during electrolytic decomposition of mineralized waters. In the process of electrolysis of mineralized water, emulsified in oil, hydrogen $\mathrm{H}_{2}$ and oxygen $\mathrm{O}_{2}$, possessing high penetrating destructing capacity in liquid alkaline medium, form. Microbubbles of these gases, formed on the surface of the rod string and TS, will upset adhesion of paraffin particles and other components of ARPD, facilitating their tearing off from metal surfaces of the subsurface pumping equipment and bringing out to the wellhead by the upstream of the borehole fluid. The electrolysis process is also accompanied by local thermal influence, preventing from formation and accumulation of ARPD in the air-gas channel of TS.

Formation of the electrostatic field is advisable to use because ARPD particles have some negative electric charge [19]; therefore, for prevention of their deposition, electric interaction between these charges and the artificially created field of wells can be used on the pumping equipment.

For creation of these effects, it is possible to use so called cathode (anode) polarization, usually applied for electrochemical protection (ECP) of pipelines [20, 21]. Cathode polarization of the protected surface can be achieved by two methods: application of the external source of current (cathode protection) or artificial creation of galvanic elements, in which the protected construction (galvanic protection or protection by autonomous anodes) is a cathode. In both cases, electric current will flow in the formed electric chain.

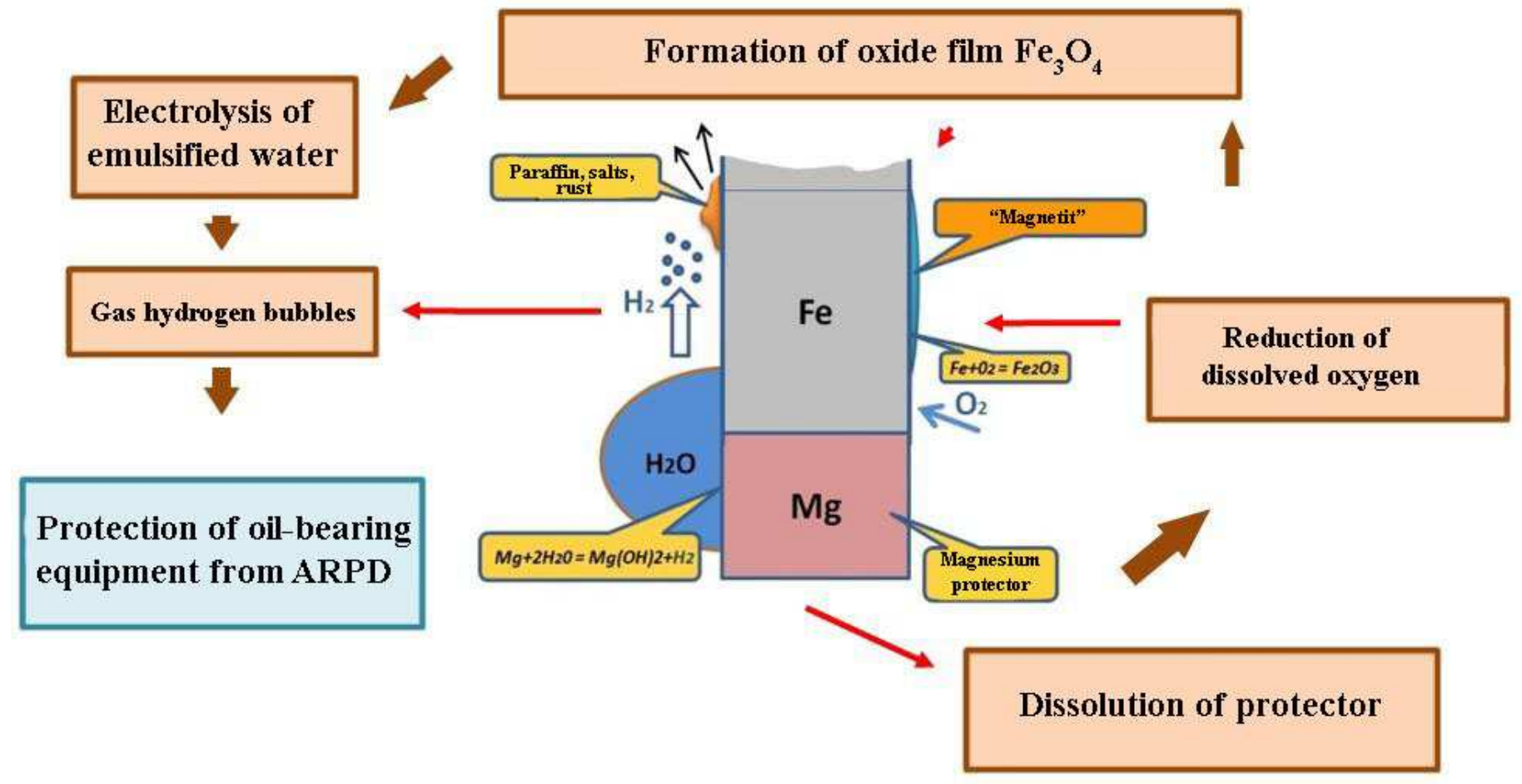

Fig. 2. Principle of proposed method of controlling ARPD. 
For creation of such electrochemical chain, an additional electrode, descended on the hoist cable and connected to the positive pole of the power source located on the wellhead, is located in TS. The negative pole is connected to TS. The passage of electric current will be accompanied by electrolysis of stratal water, emulsified in oil fluid, as a result of which hydrogen $\mathrm{H}_{2}$ and oxygen $\mathrm{O}_{2}$ will escape (Figure 2). These gas electrically-charged microbubbles, formed on colloid microadmixtures, represent a large number of additional centres of crystallisation and flotation carrying-out.

Influence of gas emission on the intensity of paraffin depositions in the general case is of ambiguous nature. In the general case, gas bubbles possess ability to float the suspended paraffin particles. When tearing off the bubble, precipitated from the borehole fluid, from the solid surface, in the beginning, the basis of the bubble contracts very slowly, and then quicker and quicker. After some time interval, the tearing off is realised abruptly. At that point, there is pressure differential between the volume phase and the zone, being under the bubble, as a result of which a part of paraffin crystals is brought to the surface, bordering on the pipe wall: they contact the wall and precipitate on it. Later the process of depositions increases progressively since paraffin possesses a hydrophobic surface. On the pipe wall, the stratum is formed from paraffin crystals and gas bubbles. The less this stratum is saturated with gas, the denser it is. Therefore, denser depositions form in the lower part of the lifting pipes, where gas bubbles are small and possess a greater force of adhesion to the paraffin crystals and pipe walls [1].

However, in case of electrolysis, oxygen and hydrogen microbubbles will form immediately in the fluid volume, exerting only floating influence on ARPD particles.

Electrolysis entails local overheating, which also enables dissolution of paraffins in oil on a certain area.

The use of electrostatic field with a polarity similar to ARPD particles allows one to additionally lower adhesion of deposition products on the surface of subsurface pumping equipment.

The running depth of the electrode corresponds to the area of the most probable beginning of paraffins deposition. It can be determined by both temperature profile of the well and special methods [22].

The use of cathode polymerization to prevent ARPD depositions allows reducing simultaneously the corrosion of oilpumping equipment owing to formation of the thin film of iron oxide $\mathrm{Fe}_{3} \mathrm{O}_{4}$, so called magnetite, on the metal. This film appears under the influence of hydroxyl ions $\mathrm{OH}-$, formed during electrolysis, which act as an oxidizer with respect to steel pipes. Round-trip operations are performed using a geophysical lifter.

\section{V.CONCLUSION}

Advantages of the mentioned method consist in the following:

- $\quad$ low cost;

\author{
possibility of application of all types of extraction; \\ simplicity of mounting; \\ environmental and industrial safety; \\ autonomy of machinery operation.
}

The tests conducted on the number of wells showed that during the control interval of time, depositions do not form or their thickness decreases to $204 \mathrm{~mm}$, i.e. almost by an order of magnitude.

Thus, application of the described technology of preventing ARPD formation with simultaneous protection of downhole equipment against corrosion in oil wells in complicated conditions of late exploitation of deposits allows increasing oil extraction, having excluded the shutdown process of exploitation wells because of depositions and, correspondingly, having increased their overhaul period. At that, no large investments are required, but all necessary measures can be realised during conducting a complex of works on capital repairs of wells.

\section{References}

[1] L.V. Ivanova, E.A. Burova, V.N. Koshelev, "Asphalt-resin-paraffin depositions during extraction, transporting and storage," Electronic science journal «Oil and Gas Engineering», №1, pp. 268-284, 2011.

[2] A.N. Ilin, Yu.M. Polishchuk, I.G. Yashchenko, "High-paraffin oils: regularities of space and time changes of their properties," Electronic science journal «Oil and Gas Engineering», 2007, №2.

[3] Yu.M. Polishchuk, I.G. Yashchenko, "Geographic regularities in the changes of physical and chemical properties of oil resources of Eurasia," Geography and natural resources, № 4, pp. 60 - 66, 2001.

[4] N.Ya. Bagautdinov, Scientific bases and technologies of influence of physical fields on hydrate-paraffin depositions in oil wells. Abstract of a thesis for Candidate of Science, Ufa, 2007.

[5] J. Carroll, Natural Gas Hydrates 2 edition. Gulf Professional Publishing, 2009, p. 288.

[6] V.P. Tronov, Mechanism of formation of resin-paraffin depositions and their control. Moscow: Nedra, 1970, p. 192

[7] M.Sh. Kayumov, V.P. Tronov, I.A. Guskov, A.A. Lipaev, "Consideration of peculiarities of formation of asphalt-resin-paraffin depositions at late stage of oil-field development,” Oil economy, № 3, pp. 48-49, 2006.

[8] B. Cranford, "New Trends in chemical control of paraffin," Drilling and production Practice, 1957, Vol. 37 (IV).

[9] M.N. Persiyantsev, Oil production in complicated conditions, Moscow: LLC «Nedra - Biznesstsentr», 2000, p. 653.

[10] E.V. Soboleva, "Analysis of conditions of formation and methods of controlling asphalt-resin-paraffin depositions in wells when extracting oil in deposits of Kama region. Herald PNIPU," Geology. Oil and gas engineering and mining, № 8, pp. $71-77,2013$.

[11] Sorokin S.A., Khavkin S.A. Peculiarities of physical and chemical mechanism of ARPD formation in wells, Drilling and oil, № 10, p. 3031, 2007.

[12] S.A. Belkina, S.N. Nagaeva, "Reasons of formation of asphalt-resinparaffin depositions in TS," Herald of Yugra State University, Issue 3 (42), pp.7-11, 2016.

[13] A.G. Malyshev, N.A. Cheremisin, G.V. Shevchenko, "Choice of optimal ways of controlling paraffin depositions," Oil economy, № 9, pp. 62 69, 1997.

[14] B.V. Karpov, V.P. Vorobev, V.T. Kazakov et al. "Prevention of paraffin depositions when extracting oil from wells in complicated conditions by using magnetic devices,” Petroleum engineering, № 12, pp. 17-18, 1996.

[15] B.V. Karpov, V.P. Vorobev, "The use of physical fields for prevention of paraffin depositions when extracting oil," Oil economy, № 7, pp. 45 47, 1997. 
[16] M.A. Fatykhov, N.Ya. Bagautdinov, "Influence of electromagnetic field on paraffin crystallisation process," Electronic Science journal «Oil and gas engineering», 2007, № 1 .

[17] A.I. Podyapolskiy, Improvement of the technology of prevention of complications when extracting high-viscosity oil. Abstract of a thesis for Candidate of Science, Ufa, 2007.

[18] Methods of controlling paraffin depositions. Retriewed from: http://proton.webtuner.ru/statii/metodyi_borbyi_s_parafinootlozheniyam i

[19] I.L. Markhasin, Physical and chemical mechanics of oil stratum, Moscow: Nedra, 1977, p. 214.
[20] A.N. Suleymenova, A.N. Krasnov, "Control and prevention of hydrate and paraffin formations," Problems of workflow automation of extracting, transporting and processing oil and gas, USOTU, 2016, pp. $146-151$.

[21] A.N. Suleymanova, A.N. Krasnov, "Peculiarities of paraffination of pumping equipment of stripped wells equipped with USSHN," Problems of workflow automation of extracting, transporting and processing oil and gas, 2017, pp. $120-125$.

[22] V.V. Misnik, R.M. Galikeev, "Method of predicting depth of formation of asphalt-resin-paraffin depositions in wells," Electronic science journal «Oil and gas engineering», №6, pp. 345-349, 2011. 